\title{
Analysis of Fuel Cells Utilizing Mixed Reality and IoT Achievements
}

\author{
Burkhard Hoppenstedt ${ }^{1}$, Michael Schmid ${ }^{2}$, Klaus Kammerer ${ }^{1}$, Joachim \\ Scholta $^{2}$, Manfred Reichert ${ }^{1}$, and Rüdiger Pryss ${ }^{1}$ \\ 1 Institute of Databases and Information Systems, Ulm University, Ulm, Germany \\ 2 Zentrum für Sonnenenergie- und Wasserstoff-Forschung Baden-Württemberg, Ulm, \\ Germany \\ burkhard.hoppenstedt@uni-ulm.de
}

\begin{abstract}
Recent advances in the development of smart glasses enable new interaction patterns in an industrial context. In the field of Mixed Reality, in which the real world and virtual objects fuse, new developments allow for advanced procedures of condition monitoring. Hereby, the smart glasses serve as a mobile display and inspection station. In this work, we focus on the applicability of Mixed Reality to monitor data of the spatially resolved current density distribution of a fuel cell. To be more specific, we implemented an IoT approach based on the Message Queuing Telemetry Transport protocol (MQTT) to enable the aforementioned monitoring. The realized solution, in turn, provides a live monitoring as well as an overview feature.
\end{abstract}

Keywords: Fuel Cells · Mixed Reality $\cdot$ IoT $\cdot$ MQTT

\section{Introduction}

In the context of the industrial internet of things (IIoT), also denoted as Industry 4.0 [18], the collection of sensor values becomes more and more crucial. These values are then used, e.g., for condition monitoring [23], process control [11], or advanced analytics (e.g., Predictive Maintenance [14]). The overall goal of a production setting connected through sensors is to increase the production efficiency by a) reducing downtimes through predictive methods, b) increasing the production transparency to discover bottlenecks, and c) enabling data-driven approaches for a self-diagnostics plant. Hereby, machine communication protocols, such as Open Platform Communications Unified Architecture (OPC UA) [13] or the Message Queuing Telemetry Transport protocol (MQTT) [16], are an essential part to exchange data in the needed distributed architectures [4]. These protocols implement features to ensure the guaranteed delivery of messages and required encryption needs. In this work, an IoT approach based on (1) the MQTT communication protocol and (2) the Microsoft HoloLens smart glass was realized to test its feasibility for the monitoring of current density distribution data of a fuel cell. 
Research into alternative energy sources is particularly important nowadays as the impact of greenhouse gases on the environment through the use of fossil fuels continues to increase as these resources become more and more scarce [3]. One way to overcome these problems could be the use of fuel cells and the expansion of the hydrogen infrastructure. Such energy conversion devices generate electricity using hydrogen and oxygen in an electrochemical process for which water is the only remaining waste product [2]. Therefore, in recent years, a variety of scientific research has been conducted to optimize fuel cells and minimize their manufacturing costs [22].

In our use case, the fuel cell represents a machine that delivers sensor values, whereas a HoloLens is the monitoring application. We connect these two devices via the MQTT protocol for a quick and trustworthy connection. The connection allows to supervise the progress of the fuel cell's sensor values as well as to automatically generated alarms, which, in turn, can be sent to various recipients (e.g., process control staff).

The remainder of the paper is structured as follows: in Section 2 related work is discussed, while Section 3 introduces the backgrounds on fuel cells, Mixed Reality, and the MQTT protocol. In Section 4, the developed prototype is presented, in which the data set, the Graphical User Interface (GUI), and the backend system are presented. Threats to validity are presented in Section 5, whereas Section 6 concludes the paper with a summary and an outlook.

\section{Related Work}

The first part of the related work refers to fuel cells. According to the United States Department of Energy (DOE) [7], fuel cells with polymer electrolyte membranes (PEMs) have been developed for use in automobiles since the late 1980's and steady progress has been made to date. Fundamental studies of electrochemical properties are particularly important for improving PEM fuel cells as they can generate current density distribution inhomogeneities due to different reactions and activities in the active cell region. These are also influenced by parameters such as temperature and humidity of the membrane and have a fundamental influence on the life cycle and performance of a fuel cell. By visualizing the current density distribution within the fuel cell as shown in Fig. 1, corresponding information can be obtained [8]. Concerning the second part of related work, augmented reality is used in various use cases to monitor aspects of the real world. In [10], 3D models are compared to real world objections for the purpose of construction supervision. Wireless sensor networks, in turn, are monitored by [9] using an augmented reality interface. The HoloLens, which represents a smart glass of the category Mixed Reality, is often utilized in the medical context (cf. [17] or [15]). However, to the best of our knowledge, a combination of technologies as shown in this work, has not been presented in other works so far. 


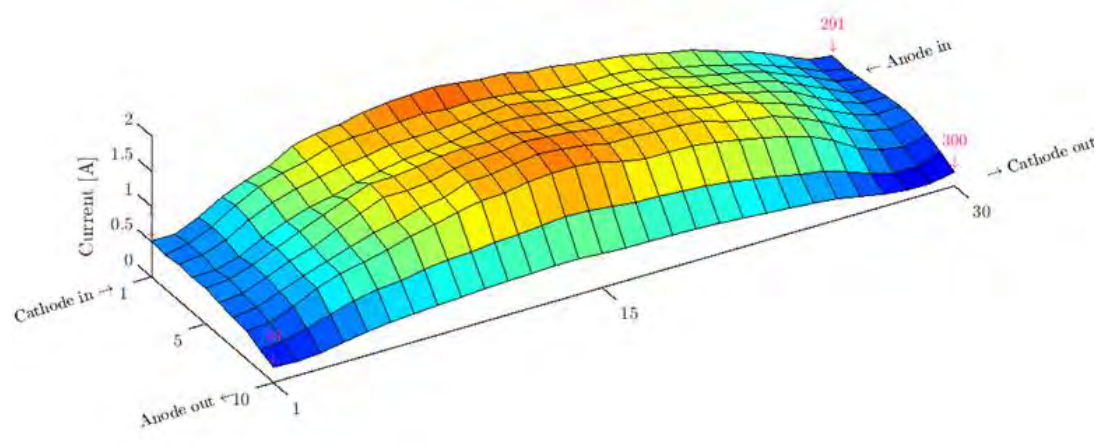

Fig. 1. Visualization of the current density distribution inside a fuel cell

\section{Fundamentals}

\subsection{Fuel Cells}

Hydrogen $\left(\mathrm{H}_{2}\right)$ is introduced on the anode side and air containing oxygen $\left(\mathrm{O}_{2}\right)$ on the cathode side. At the anode, the molecular hydrogen is split into hydrogen nuclei $\left(\mathrm{H}^{+}\right)$, also called protons and electrons $\left(\mathrm{e}^{-}\right)$, with the help of a catalyst. The protons migrate through the electrolyte membrane - which is permeable only to them - to the cathode side. The electrons travel from the anode through an electrical conductor to the cathode. The resulting current flow, in turn, can be exploited. On the cathode side, two electrons reduce oxygen which then combines with two $\mathrm{H}^{+}$-ions to form water $\left(\mathrm{H}_{2} \mathrm{O}\right)$ [5], as shown in Equation 1. This electrochemical process is schematically shown in Fig. 2.

$$
2 \mathrm{H}^{+}+\mathrm{O}_{2}^{-} \longrightarrow \mathrm{H}_{2} \mathrm{O}
$$

\subsection{Mixed Reality}

The HoloLens is a device to realize mixed-reality applications. Mixed Reality is known to have the highest intersection of reality and virtual environment of all augmented reality approaches [20] due to a concept named spatial mapping. This procedure creates a model of the environment in the augmented reality device. Therefore, interactions of holograms and real-world objects become possible. Mixed Reality is basically used to display 3D models (1) for which a real-world model would be too large or small (e.g., in the domains automotive or architecture), (2) in medical use cases (assistance during a surgery), or (3) in industrial maintenance support setting. The HoloLens is equipped with various cameras and sensors, such as a depth sensor, a RGB camera, and an ambient light sensor. The holograms can be anchored to real-life objects, but infinite projections are 


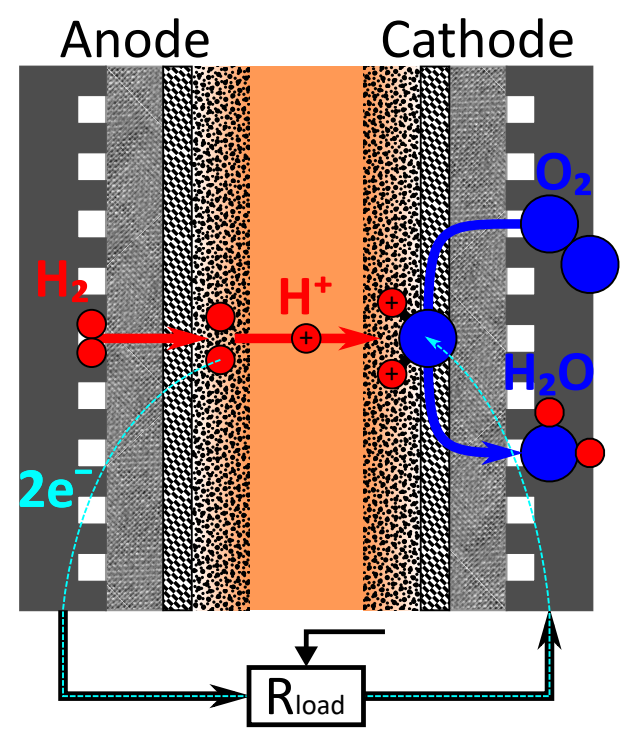

Fig. 2. Schematic of the electrochemical reaction inside a fuel cell

not possible, neither to the distance nor to the proximity. With a weight of $579 \mathrm{~g}$, the HoloLens should not be used for a long period of time due to an unnatural head positioning. A HoloLens case study [25] found out that the heavy weight of the device degrades the user's comfort level. Finally, in an intensive use case, the battery lasts for about 2.5 hours, which also inhibits a long-time usage.

\subsection{Message Queuing Telemetry Transport Protocol}

The Message Queuing Telemetry Transport protocol (MQTT) is a light-weight machine to machine communication protocol. It uses a publish-subscribe [6] pattern, including the use of topics. According to [16], publish/subscribe systems are wide-spread in distributed computing. Hereby, a topic can be considered as a black board for messages. Subscribers are informed about changes to these topics and new messages (e.g., sensor values) can be pushed to these topics. A distribution server, denoted as broker, is responsible to forward messages to subscribed clients. MQTT offers a Quality of Service (QoS) level [19], for which the delivery of a specific message is guaranteed at most once, at least once, or exactly once. MQTT has, in contrast to OPC UA, no semantic structure and can therefore transport any kind of message. All these mechanisms make MQTT a suitable communication protocol for IoT use cases. 


\section{Prototype}

The realized prototype shall allow for the monitoring of the current density distribution of a fuel cell in Mixed Reality. More specifically, the Microsoft HoloLens is used as MQTT client to display the current state of the fuel cell. The user, in turn, shall be enabled to interact with the holographic visualization through the MQTT interface. Following this, for example, the values of the fuel cell can be monitored and evaluated in real time. As a first step of the developed prototype, the fuel cell was digitized using a 3D modeling tool (i.e., Blender, see also Fig. $3)$. Hereby, the arrows are animated to indicate input and output of the fuel cell gases. A cell grid represents all measuring points in the fuel cell. As a next step, the blender model can be attached with interaction logic. Therefore, we implemented a tap to place method, so that the model can be placed anywhere in the real world.

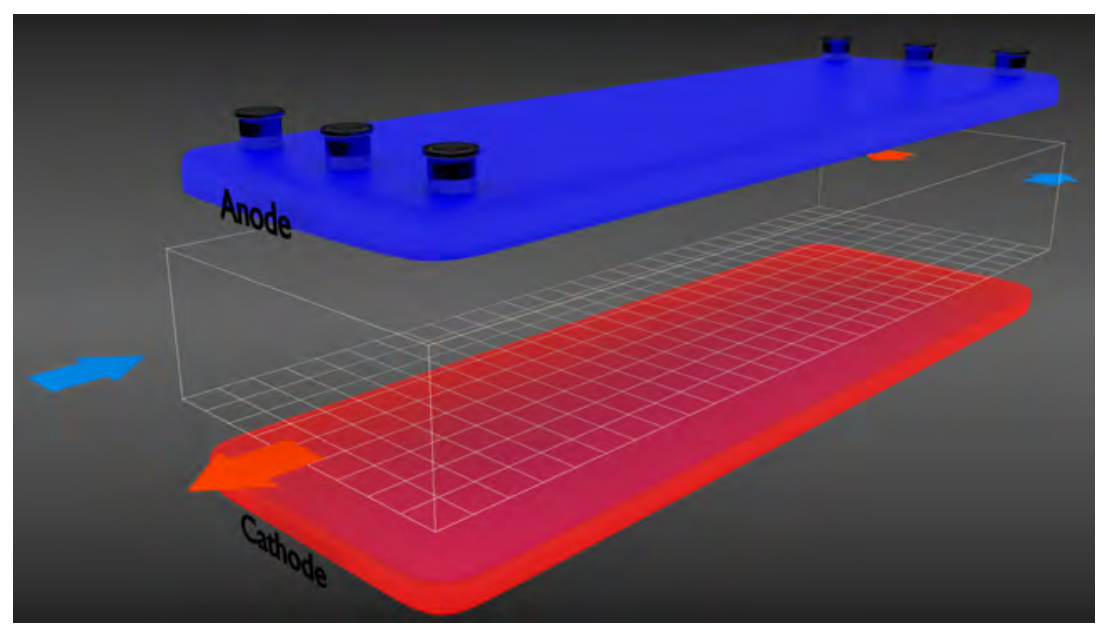

Fig. 3. 3D rendering of the fuel cell end plates with space for the current density distribution values for spatial visualization with the HoloLens device

Then, the values of the fuel cell are sent to the model for monitoring. We implemented the use cases live monitoring and loading of a data set. The main difference constitutes a possible replay and change of playing speed for the second use case. The control for the replay is provided by MQTT. As we integrated a MQTT client into the HoloLens application, MQTT can be used as a remote control to set the current frame or frame rate. Using the replay mode, it is possible to get a quick overview of the temporal behavior by viewing the sensor values in a time-lapse mode. In contrast, when using the live mode, it is possible to be alerted via a sound or sending the alert to any IoT device that can implement 
the MQTT protocol (e.g., smartphones, machines, or computers). The realized architecture of the prototype is shown in Fig. 4

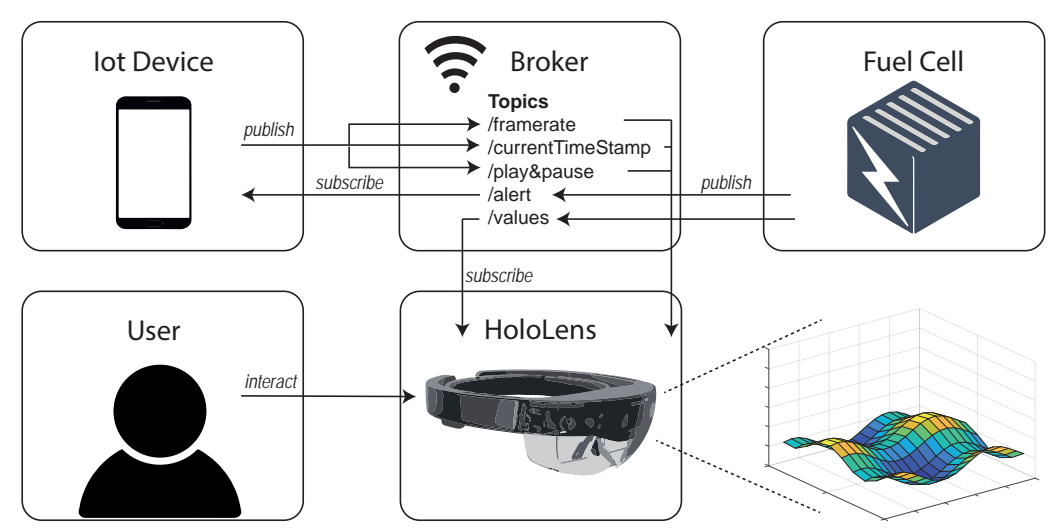

Fig. 4. Prototype Architecture

\section{$5 \quad$ Threats to Validity}

The following limitations need to be considered for the work at hand. First, the weight of the HoloLens smart glass cannot be neglected. Intensive use might cause headaches or dizziness. Second, the further connectivity of the HoloLens might be a problem. The options for data science analyses in Mixed Reality are not sufficiently evolved so far. However, the possibilities of data analytics in augmented reality, denoted as immersive analytics, are more and more investigated [12]. Third, a general problem of distributed systems is the network security [26] and network stability. In our approach, we solely rely on the connectivity and security features implemented by MQTT. Finally, new interaction methods, provided by the HoloLens, also result in new challenges. When using the HoloLens via voice commands, it is essential to be aware of a user bias. For the voice commands, studies have shown that speech recognition performs worse for women compared to men (cf. [24], [21]). Despite these limitations, the strength of our prototype was that we combined a fuel cell, an IoT protocol, and a contemporary smart glass to an interactive visualization approach.

\section{Summary and Outlook}

We presented a prototype for the monitoring of the current density distribution of a fuel cell in Mixed Reality. The architecture incorporates an IoT message 
protocol (MQTT) for a light-weight communication to any device that supports this protocol. The Microsoft HoloLens, which represents a mixed-reality smart glass, is used as MQTT client to display the current state of the fuel cell. The user can interact with the holographic visualization using voice commands or by sending commands via the MQTT interface. The values of the fuel cell can be either inspected in real time or by using a preloaded data set. The latter offers a quick inspection, e.g., by using a time-lapse function. Currently, the field of view for the HoloLens is limited to a small window. However, upcoming types of mixed-reality glasses will fix this limitation and offer new user interaction patterns. Moreover, the analytic part of our approach is currently limited to alerts. By including stream analytic approaches [1], we could provide a more powerful online analytic tool. In the presented approach, solely the current density distribution was used. However, the spatially resolved visualization of the temperature and humidity would be also promising targets. Moreover, other important values may be presented in an interactive head-up display in order to keep an eye on the condition of the fuel cell in an even more efficient manner. On top of this, it is conceivable to adapt the operating parameters by means of gestures or voice controls. Altogether, this work has shown that Mixed Reality can play an important role in different domains that are less considered so far.

\section{Acknowledgements}

The authors want to thank the German Federal Ministry for Economic Affairs and Energy for funding part of the presented work within the project SoHMuSDaSS (FKZ: 03ET6057C).

\section{References}

1. Andrade, H.C., Gedik, B., Turaga, D.S.: Fundamentals of stream processing: application design, systems, and analytics. Cambridge University Press (2014)

2. de Beer, C., Barendse, P.S., Pillay, P., Bullecks, B., Rengaswamy, R.: Classification of High-Temperature PEM Fuel Cell Degradation Mechanisms Using Equivalent Circuits. IEEE Transactions on Industrial Electronics 62(8), 5265-5274 (aug 2015)

3. Chandan, A., Hattenberger, M., El-kharouf, A., Du, S., Dhir, A., Self, V., Pollet, B.G., Ingram, A., Bujalski, W.: High temperature (HT) polymer electrolyte membrane fuel cells (PEMFC) A review. Journal of Power Sources 231, 264-278 (jun 2013)

4. Datta, S.K., Bonnet, C., Nikaein, N.: An iot gateway centric architecture to provide novel $\mathrm{m} 2 \mathrm{~m}$ services. In: Internet of Things (WF-IoT), 2014 IEEE World Forum on. pp. 514-519. IEEE (2014)

5. Ehret, O.: Wasserstoff und Brennstoffzellen: Antworten auf wichtige Fragen. NOW GmbH - Nationale Organisation Wasserstoff- und Brennstoffzellentechnologie, Berlin (2018)

6. Eugster, P.T., Felber, P.A., Guerraoui, R., Kermarrec, A.M.: The many faces of publish/subscribe. ACM computing surveys 35(2), 114-131 (2003)

7. Fuel Cell Technologies Office: Multi-Year Research, Development, and Demonstration Plan. Tech. rep., U.S. Department of Energy (2012) 
8. Geske, M., Heuer, M., Heideck, G., Styczynski, Z.A.: Current density distribution mapping in PEM fuel cells as an instrument for operational measurements. Energies 3(4), 770-783 (2010)

9. Goldsmith, D., Liarokapis, F., Malone, G., Kemp, J.: Augmented reality environmental monitoring using wireless sensor networks. In: 12th International Conference Information Visualisation. pp. 539-544. IEEE (2008)

10. Golparvar-Fard, M., Peña-Mora, F., Savarese, S.: D4ar-a 4-dimensional augmented reality model for automating construction progress monitoring data collection, processing and communication. Journal of information technology in construction 14(13), 129-153 (2009)

11. Gonzaga, J., Meleiro, L.A.C., Kiang, C., Maciel Filho, R.: Ann-based soft-sensor for real-time process monitoring and control of an industrial polymerization process. Computers \& chemical engineering 33(1), 43-49 (2009)

12. Gracia, A., González, S., Robles, V., Menasalvas, E., Von Landesberger, T.: New insights into the suitability of the third dimension for visualizing multivariate/multidimensional data: A study based on loss of quality quantification. Information Visualization 15(1), 3-30 (2016)

13. Hannelius, T., Salmenpera, M., Kuikka, S.: Roadmap to adopting opc ua. In: 6th IEEE International Conference on Industrial Informatics. pp. 756-761. IEEE (2008)

14. Hoppenstedt, B., Pryss, R., Stelzer, B., Meyer-Brötz, F., Kammerer, K., Treß, A., Reichert, M.: Techniques and emerging trends for state of the art equipment maintenance systemsa bibliometric analysis. Applied Sciences 8, 1-29 (2018)

15. Hoppenstedt, B., Schneider, C., Pryss, R., Schlee, W., Probst, T., Neff, P., Simoes, J., Treß, A., Reichert, M.: Holoview: Exploring patient data in mixed reality. In: TRI / TINNET Conference 2018 (2018)

16. Hunkeler, U., Truong, H.L., Stanford-Clark, A.: Mqtt-sa publish/subscribe protocol for wireless sensor networks. In: 3rd international conference on Communication systems software and middleware and workshops. pp. 791-798. IEEE (2008)

17. Hurter, C., McDuff, D.: Cardiolens: remote physiological monitoring in a mixed reality environment. In: ACM SIGGRAPH 2017 Emerging Technologies. p. 6. ACM (2017)

18. Lasi, H., Fettke, P., Kemper, H.G., Feld, T., Hoffmann, M.: Industry 4.0. Business \& Information Systems Engineering 6(4), 239-242 (2014)

19. Lee, S., Kim, H., Hong, D.k., Ju, H.: Correlation analysis of mqtt loss and delay according to qos level. In: International Conference on Information Networking. pp. 714-717. IEEE (2013)

20. Milgram, P., Takemura, H., Utsumi, A., Kishino, F.: Augmented reality: A class of displays on the reality-virtuality continuum. In: Telemanipulator and telepresence technologies. vol. 2351, pp. 282-293. International Society for Optics and Photonics (1995)

21. Nicol, A., Casey, C., MacFarlane, S.: Children are ready for speech technology-but is the technology ready for them. Interaction Design and Children, Eindhoven, The Netherlands (2002)

22. Orfanidi, A.: Electrocatalytic Investigation of High Temperature PEM Fuel Cells Alin Orfanidi (December) (2014)

23. Rangwala, S., Dornfeld, D.: Sensor integration using neural networks for intelligent tool condition monitoring. Journal of Engineering for Industry 112(3), 219-228 (1990) 
24. Rodger, J.A., Pendharkar, P.C.: A field study of the impact of gender and user's technical experience on the performance of voice-activated medical tracking application. International Journal of Human-Computer Studies 60(5-6), 529-544 (2004)

25. Zhang, L., Dong, H., El Saddik, A.: Towards a qoe model to evaluate holographic augmented reality devices: A hololens case study. IEEE MultiMedia (2018)

26. Zhang, Z.K., Cho, M.C.Y., Wang, C.W., Hsu, C.W., Chen, C.K., Shieh, S.: Iot security: ongoing challenges and research opportunities. In: 7th International Conference on Service-Oriented Computing and Applications. pp. 230-234. IEEE (2014) 